\title{
Immunotherapy in Advanced Prostate Cancer: Letter to the Editor
}

\author{
Gilles Plourde* \\ Associate Professor Department of Clinical Pharmacology and Physiology, Faculty of Medicine, University of Montreal, Canada
}

Submission: November 07, 2017; Published: November 16, 2017

*Corresponding author: Gilles Plourde MD,PhD Associate Professor Department of Clinical Pharmacology and Physiology, Faculty of Medicine, University of Montreal, Montreal, Quebec, Canada; Email: gilles.plourde@hc-sc.gc.ca; drgplourde@gmail.com

\section{Introduction}

Prostate cancer (PC) accounts for about 1 in 7 to 1 in 5 new case (incidence) of cancer in US and Canada and, despite the recent improvements, prostate cancer still causes more than 26,000 deaths per year [1-3]. A reduction of immune activity seems to play a prominent role in the development of cancer [45]. It was found that cancerous cells can escape T-cell responses to tumor-associated antigens by multiple mechanisms. The role of active immunotherapy is to stimulate the immune response against cancer cells. Prostate cancer is not ignored by the immune system, as evidenced by the presence of tumor infiltrating lymphocytes in the prostatic tissue affected by this cancer. This characteristic permits prostate cancer cellsto become suitable for immunotherapy [5]. The presence of welldefined antigens, largely limited to prostate tissue, permits these cells to be targeted by the immune system without the risk of systemic autoimmune reactions $[5,6]$.

Active immunotherapy in prostate cancer can be conducted using multiple strategies such as dendrites' cells, whole-cell vaccines, viral vectors, DNA-based and peptide-based agents, as well as immunostimulatory agents. The only FDA-authorised immunotherapy for prostate cancer is the dendritic-cell-based vaccine named Sipuleucel-T, which has an advantage in overall survival, but not in progression-free survival $[5,6]$. This product was not authorised in Canada or Europe. With the case report presented below and in order to be able to respond adequately to the question raised by the patients, I will briefly discuss the clinical development in the field of immunotherapy for the treatment of advanced prostate cancer. Considering that in the context of a short communication, it is almost impossible to cover all the information available on this issue, here I discussed particularly relevant information for counselling patients with advanced prostate cancer.

\section{Case Report}

A 61 year-old patient known for a poorly differentiated and aggressivestage IV prostate cancer came to your office. He was treated with Zoladex and Casodex for 3 years. Recently, Casodex was interrupted because his PSA level has started to increase significantly and he was then put on Zytiga, a second line hormone therapy in combination with Zoladex and prednisone. He also has bone metastasis for which he received Xgeva. He was looking at the medical literature and from what he has read; he is highly excited by the development in the field ofimmunotherapy and his suggesting that immunotherapy must probably be an appropriate option for him. For further information on the patient presented in this case report, please consult two recent publications from Dr. Plourde in the same journal $[7,8]$.

The first question asked by the patient is why we can fail or become resistant to androgen deprivation therapy (ADT)

Androgen deprivation therapy (ADT) is currently the main treatment for advanced PC. However, eventually, most patients progress to a condition known as castration-resistant prostate cancer (CRPC) or hormone-resistant which is characterized by a lack of response to ADT. Although new androgen receptor signaling (ARS) inhibitors and chemotherapeutic agents have been introduced to overcome resistance to ADT, patients still become resistant to these agents [9]. The majority of treatments that are currently used in clinical practice to treat patients with PC modulate the ARS pathway. However, the increased 
heterogeneity and genomic variability of advanced PC cells are frequently responsible for the resistance to treatment [9].

Many pathways, both androgen receptors (AR) related and non-AR related, may play a role to the progression of hormone resistance [5,6]. In many instances, AR-independent pathways are involved in the growth of aggressive tumor cells with high metastatic potential. Many non-AR-directed drugs have been tested in hormone resistant prostate cancer, mostly as monotherapy and these studies provide conflicting results $[5,6]$. As discussed in recent article predictive biomarkers of response to treatment are therefore needed to increase the success of future trials, and to properly select patients that would most likely benefit from these non-AR-targeted therapies [7]. In addition, studies evaluating the combination of ARS modulators or chemotherapy with molecules that target AR-independent pathways may provide new treatment options for advanced PC [9] (See below).

\section{The Second Questions ask by the Patient is Why We Should Consider Immunotherapy}

As you now know, immunotherapy modulates and reinforce the patient own immune responses against cancer in general and must probably against advanced prostate cancer $[5,6]$. As briefly explained in the introduction, neoplastic cells naturally can escape from the control of the immune system, and the main goal of immune therapy is to get this short- and longterm control back. Previous studies in advanced melanoma and lung cancer suggest a great potential of immunotherapy as an approach for other tumors' treatment including prostate cancer $[5,6]$ with less side effects than chemotherapy. As mentioned earlier PC was the first neoplasm in which a specific vaccine that significantly improved PC survival. Furthermore, there is a strong potential for synergistic combinations of immunotherapy with conventional cancer treatments including prostate cancer [10-13].

Then the Patient Asks Why Immunotherapy Could Be Not That Performant for His Advanced Prostate Cancer Treatment

Strategies that use checkpoint inhibitors targeting PD1/ PDL1 have been successful in treating other malignancies, but unfortunately were not successful in the treatment of prostate cancer $[10,11]$. We can hypothesis that the resistance to checkpoint inhibition can be overcome in prostate cancer through targeting of myeloid derived suppressor cells (MDSCs), which are involved in suppressing $\mathrm{T}$ cell responses, which protects the cancer from the immune mediated action resulting in cancer cell destruction [10,11]. Importantly MDSCs are prevalent in metastatic prostate cancers. However, neither checkpoint inhibition nor MDSC targeting provides significant effect in mouse models of prostate cancer. However when combined, the therapeutic effect is synergistic and can be more efficient [10], but this still need to be studied.
Then the Patient Suggests that If We Combine Two Immune Therapies Whether Or Not Our Chance of Success In Treating His Advanced Prostate Cancer Would Be Increased

As just discussed, we can suggest that a combined therapeutic approach may provide a more effective immunotherapy in patients with advanced prostate cancer. In fact, it was recently discovered that the combination of 2 immunotherapy drugs could help increase the response of the immune system against advanced prostate cancer cells. However, the existence on a human tumor of a third brake called VISTA that may inhibit immune response was found [4]. Recently, in March 2017, Gao J et al. [4] performed a clinical trial in patients with advanced prostate cancer combining 2 immunotherapy drugs targeting specific brakes on the immune system. This initial study, published in Nature Medicine, has explored whether they could increase immune cell infiltration of tumors by combining the ADT drug leuprolide with 2 rounds of the checkpoint inhibitor ipilimumab before surgery in patients with locally advanced prostate cancer. Importantly, genomic and immune analysis of the surgically removed tumors demonstrated high levels of penetration of the tumors by activated $\mathrm{T}$ cells. This study also indicated increased levels of immune-suppressing PD-L1 and VISTA. PD-L1 is known to interact with the immune checkpoint PD- 1 on $T$ cells, thus activating PD- 1 to block the T cell. Gao J et al. [4] mentioned that driving $\mathrm{T}$ cells into the tumors would be the first step, but the next step would be to block PD-L1 and VISTA.

These results have been applied in an immunotherapy combination clinical trial that use ipilimumab to bring $\mathrm{T}$ cells into the tumour and the PD-1 inhibitor nivolumab to attack the PD-L1/PD-1 response [11]. The trial has enrolled 90 patients at 9 centers nationally. Although 6 therapies have been approved to treat patients with metastatic, hormone-resistant prostate cancer, none has provided a durable response [11]. The article published in Nature Medicine strongly suggest the importance of studying immune response longitudinally and that simply observing it at one point in time does not reflect what is occurring because the immune system changes so quickly [4].

Then the patient suggests that if we combine immunotherapy with conventional or other treatments whether or not our chance of success in treating his advanced prostate cancer would increase.

ADT is the main therapy for prostate cancer, and agents targeting the AR pathway continue to be developed such as Zytiga (abiraterone) which is a second line hormone therapy that the patient is currently taking [8]. Because ADT has immmunostimulatory effects as well as direct antitumor effects, AR-targeted therapies could be combined with other anticancer therapies, including immunotherapies. A recent study evaluating whether antigen-specific mechanism of resistance to ADT [12] may result in enhanced AR-specific T-cell immune 
recognition, and whether this might be strategically combined with an antitumor vaccine targeting the AR has been performed. ADT increased AR expression in human and murine prostate tumor cells in vitro and in vivo [12].

This increased expression persisted over time. The increased AR expression was associated with recognition and cytolytic activity by AR-specific T cells. Furthermore, ADT combined with vaccination, specifically a DNA vaccine encoding the ligandbinding domain of the AR, led to improved antitumor responses as measured by tumor volumes and delays in the emergence of CRPC tumors in two murine prostate cancer models (Myc$\mathrm{CaP}$ and prostate-specific PTEN-deficient mice) [11]. Together, these data suggest that ADT combined with AR-directed immunotherapy targets a major mechanism of resistance, over expression of the AR. This combination may be more effective than ADT combined with other immunotherapeutic approaches $[12,13]$.

Several completed and ongoing studies have shown that the combination of cancer vaccines or checkpoint inhibitors with different immunotherapeutic agents, hormonal therapy (enzalutamide), radiotherapy (radium 223), DNA-damaging agents (olaparib), or chemotherapy (docetaxel) can enhance immune responses and induce more dramatic, long-lasting clinical responses without significant toxicity [13]. The goal of prostate cancer immunotherapy does not have to be complete eradication of the advanced disease but rather the return to an immunologic equilibrium with an indolent disease state $[12,13]$. In addition to determining the optimal combination of treatment regimens, efforts are also ongoing to discover biomarkers of immune response [7]. With this concerted efforts, the use of immunotherapy in the treatment of prostate cancer seems more promising.

\section{Discussion}

Immunotherapy continues to provide new and promising tools for fighting cancer and scientists are exploring these molecules for multiple cancers [14]. However, numerous challenges remain before it becomes applicable in human patients with advanced prostate cancer. Fortunately, immunotherapy is giving to the scientific community new options for patients, and some are becoming promising treatments [15]. There has been amazing progress in the last 10 years, but we're still in the very early days in the development of this approach. Again before this treatment becomes available in patients with advanced prostate cancer, much more research needs to be done and currently these therapies are far from being a cure [6]. At this moment, we are still trying to identify the patients for which these available therapies will be sufficient for the long-term control of their cancer, but scientists are still trying to understand what it is about the patients and their tumours' that make the drugs efficient, and why others do not respond at all [11].

As mentioned these therapies are considered less toxic than many types of chemotherapy because it involves the body's own immune system to fight cancer. The significant success of immune checkpoint inhibitors in shrinking advanced melanomas led to intensified efforts to apply the therapy to a variety of other cancers including prostate cancer [15]. In 2016, the FDA, the EMA and Health Canada have approved immune therapies for advanced forms of lung, kidney, bladder, and head and neck cancer as well as Hodgkin lymphoma; this resulted in extending the survival for some groups of patients with the above cancers. The pace of approvals and new research has continued this year. Recently, the FDA, EMA and Health Canada granted accelerated marketing authorisation to the immunotherapy pembrolizumab, a programmed cell death protein 1 (PD-1) inhibitor, for patients with solid tumours [16].

The therapy is approved for adult and paediatric patients whose cancer has progressed despite prior treatment and who have no other treatment options. It the first time that the FDA, Health Canada and the EMA have approved a cancer treatment based solely on the presence of a genetic feature (BRAF V600 mutant) in a tumor; this indicate a new step toward personalized medicine in the treatment of cancer [11]. Pembrolizumab also was shown in a clinical trial to extend survival by 2 months for patients with non-small-cell lung cancer compared with standard chemotherapy [16]. And within that group of patients, those with higher levels of PD-L1 had a median survival of 15 months compared with 8 months in the control group. Another PD-1 checkpoint inhibitor, atezolizumab (Tecentriq) was the first such inhibitor to being authorised for some patients with advanced bladder cancer, as well as for patients with previously treated NSCLC [17].

Some of the biggest challenges in the field of immunotherapy lie in determining how and why patients develop resistance, as well as which specific groups respond to the therapy and why. The majority of immune therapies fall in the $25 \%$ to $30 \%$ patient response range [11]. Another major strategy in addition to immune checkpoint inhibitors is the T-cell based therapy, specifically chimeric antigen receptor (CAR) T cells, a form of adoptive cell transfer [8]. The approach involves drawing blood from patients and separating out the T cells. Using a killed virus, the $\mathrm{T}$ cells then are genetically engineered to produce CARs on their surface. These receptors allow the $\mathrm{T}$ cells to recognize and attach to an antigen on the tumour cells. They then are infused back into the patient to multiply and recognize and destroy cancer cells. CAR T-cell therapy has been shown to be effective in patients with advanced blood cancers. For more information about the CART-cell, please look at the recent letter to editor from Dr. Plourde on this issue [8]. However, it still is unclear whether this method will be successful in patients with solid tumors. As discussed above, the efficacy of immunotherapy has been more difficult to demonstrate in prostate than in other tumor types. This could be explained by the fact that a lot of prostate cancer involves the bone, as in our patient, which makes some scientists questioning if there is something in the bone structure that makes immunotherapy more challenging [6]. Unfortunately, 
currently we cannot reassure our patient about the use of immunotherapy for the treatment of his stage IV prostate cancer. However, a large number of studies are currently ongoing to find a response to this question, but patients will have to wait at least for few years before it becomes available to them. It is possible that this treatment becomes available as mono therapy but most probably in combination therapy.

\section{References}

1. Canadian Cancer Society, What is prostate cancer (2017).

2. American Cancer Society, Key Statistics for Prostate Cancer (2017).

3. Siegel RL, Miller KD, Jemal A (2016) Cancer statistics. CA Cancer J Clin 66(1): 7-30.

4. Gao J, Ward JF, Pettaway CA, Shi LZ, Subudhi SK, et al. (2017) VISTA is an inhibitory immune checkpoint that is increased after ipilimumab therapy in patients with prostate cancer. Nat Med 23(5): 551-555.

5. Janiczek M, Szylberg L, Kasperska A, Kowalewski A, Parol M, et al. (2017) Immunotherapy as a Promising Treatment for Prostate Cancer: A Systematic Review. Journal of Immunology Research 2017: 6.

6. Bilusic M, Madan RA, and Gulley JL (2017) Immunotherapy of Prostate Cancer: Facts and Hopes. Clin Cancer Res 23(22): 1-7.

7. Archambault $W$ and Plourde G (2017) Biomarkers in Advanced Prostate Cancer. Mini Review. J of Pharmacol\&Clin Res 3(5): 1-9.
8. Plourde G (2017) Gene Therapy in Advanced Prostate Cancer: Letter to the Editor. J of Pharmacol \& Clin Res 4(1): 1-3.

9. Cattrinia C, Zanardia E, Vallomea G, Cavo A, Cerbone L, et al. (2017) Targeting androgen-independent pathways: new chances for patients with prostate cancer? Critical Reviews in Oncology / Hematology 118: 42-53.

10. Lu X, Horner JW, Paul E, Shang X, Troncoso P, et al. (2017) Effective Combinatorial Immunotherapy for Castration-Resistant Prostate Cancer. Nature 543: 728-732.

11. (2017) Cancer Scope: Cancer Volume 123(21).

12. Gill MD, Agarwal N (2017) Cancer immunotherapy: A paradigm shift in the treatment of advanced urologic cancers. Urologic Oncology: Seminars and Original Investigations.

13. Wei Xiao X, Ko Eric C, Ryan, Charles J (2017) Treatment strategies in low-volume metastatic castration-resistant prostate cancer. Current Opinion in Urology. 27(6): 596-603.

14. Olson BM, Gamat M, Seliski J, Sawicki T, Jeffery J, et al. (2017) Prostate cancer cells express more androgen receptor (AR) following androgen deprivation, improving recognition by AR-specific $\mathrm{T}$ cells. Cancer Immunol Res. Oct 19.

15. Botta GP, GranowiczE, Costantini C (2017) Advances on immunotherapy in genitourinary and renal cell carcinoma. Transl Cancer Res 6(1): 1729.

16. Keytruda: Pembrolizumab: Product Monograh (2017).

17. Tecentriq (atezolizumab) Product Monograph (2017).

\section{Your next submission with Juniper Publishers will reach you the below assets}

- Quality Editorial service

- Swift Peer Review

- Reprints availability

- E-prints Service

- Manuscript Podcast for convenient understanding

- Global attainment for your research

- Manuscript accessibility in different formats

( Pdf, E-pub, Full Text, Audio)

- Unceasing customer service

Track the below URL for one-step submission https://juniperpublishers.com/online-submission.php 